\title{
Discussion on the Creating Techniques of Watercolor Painting in the Pork
}

\author{
Liu Guoji $1^{1, *}$ \\ ${ }^{1}$ South China Institue of Software Engineering.GU, Conghua, Guangzhou, Guangdong, China \\ *Corresponding author.Email: 285739162@qq.com
}

\begin{abstract}
The Pork is a series of watercolor paintings by the author, consisted of three watercolor paintings of pork. This paper attempts to discuss the performance techniques and comprehensive application creation of watercolor tool materials through The Pork and tells the possibility of exploring the performance techniques of watercolor painting through discussion and practice on watercolor materials. With the case analysis of watercolor painting on the advantages and disadvantages, this paper explains and describes the watercolor material performance effect and performance techniques based on the existing relevant research theory, so as to enrich the watercolor performance techniques.
\end{abstract}

Keywords: the Pork, drawing creation, watercolor tool materials, steps and techniques

\section{浅谈水彩《豚肉散记》的创作与技法}

刘国基 ${ }^{1, \text { a* }}$

1'州大学华软软件学院游戏系, 从化, 广州, 广东, 中国

${ }^{2} 285739162 @ q q . c o m$

\section{摘要}

《豚肉散记》是笔者所创作的一件水彩组画作品。由三张画猪肉的水彩画构成, 因豚是猪的别名, 所 以最终起名《豚肉散记》。本文试图通过《豚肉散记》论述水彩工具材料的表现技法以及综合应用创 作。记录了通过大量的水彩材料实践探讨水彩画的表现技法可能性。通过绘制水彩画案例分析其中的 优缺点, 结合已有的相关研究理论和实践对水彩材料表现效果和表现技法进行解释和描述, 以达到能 丰富水彩画表现技法的目的。

关键词：豚肉散记，创作，水彩工具材料，步骤技法

\section{1. 前言}

水彩颜料是一种可溶于水的材料, 具有一种无光泽 的表面效果。笔者喜欢它的明亮度、直接性、色彩纯度 和清新感。水彩画的物质材料是构成绘画语言的基因, 从这个角度出发, 我们可以说, 画种的本体语言是由材 质决定的, 而材质又决定了技法。所谓材质是指画家在 作画时对绘画材料的选择并由此造就出的视觉品质, 它 是画家心灵与感觉的延伸, 也是作品最终的表达形式。 在国外绘画大师的画册《席勒作品集》 ${ }^{[11}$ 和《萨金特画 集》 ${ }^{2} \mathbf{1}$ 中, 我们能看到许多优秀的水彩画作品, 可以为 我们提供许多作画思路。在国内水彩大师的画册《王肇 民水彩画作品集》 ${ }^{\mathbf{3} \mathbf{I}}$ 和《喧器的结构: 龙虎作品》【4】 中, 我们可以看到: 多技法, 新材料, 新观念的作品层 出不穷。这足以证明：画家的审美追求和思想观念需要
工具材料与表现技法做支撑，技法与材料在水彩画中已 经扮演着非常重要的角色。本文试图在现有水彩画材料 中创作作品并展示不同水彩材料的特点以及表现技法, 并以笔者的水彩作品《豚肉散记》为例加以说明。如图 1
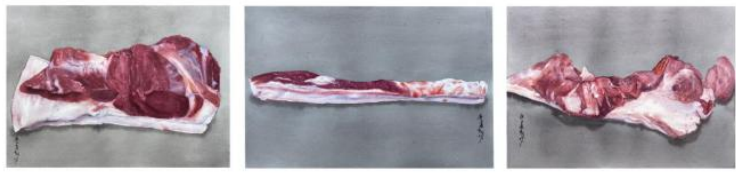

图 1 豚肉散记组画 


\section{2. 《豚肉散记》创作灵感与构思}

\section{1. 《豚肉散记》缘起}

猪又名豕、豚。因饲养简易, 又具有骨细筋少肉多 的特点, 为日常食用肉最多的一种 ${ }^{\mathbf{5 1}}$ 。自 2019 年起猪 肉价格飞涨, 也引起了笔者的极大关注, 用水彩工具材 料来表现猪肉的想法应运而生。由此选择的对象为超市 的上架猪肉, 是平头百姓所熟悉的对象, 表现这样熟悉 的对象能更好的拉近与观众的距离, 产生更好的亲和力。 猪肉本身的形与色所形成的美感, 使得笔者对对象充满 了强烈的绘画表现欲望。画一张还不足以表达笔者的全 部绘画感情, 所以画了三张组成一件组画作品的样式。 这三张猪肉组画组合在一起, 成一系列作品, 互相联系 又各自独立, 组合整体统一, 基本表达了笔者的审美趣 味与情感。

为了体现出一块不庸俗的肉的感觉, 在形式上采用 对称式构图, 这是一种能体现出严肃的、高贵的样式。 当然同时也要避免这种样式可能会带来的僵硬的、死板 的感觉, 扬长避短方是上策。背景采用平涂的灰色来祄 托出肉的红与白。背景要画的特别干净才能体现出这种 感觉, 哪怕是灰色也要灰的干净, 为此笔者作了好几种 水彩工具材料的实验案例, 后面将一进步论述这些案例 的效果。

\section{2. 《豚肉散记》呈现的风格面貌}

《豚肉散记》总的风格还是偏向写实的, 对象主体 基本形与色是按照客观对象忠实的描绘, 背景不做具体 的刻画, 但是平涂了灰底, 以便更好的祄托出主体, 为 了更好的祄托主体, 背景给人的感觉必须是平整、厚实、 干净。这样整个画面干净利落, 色彩鲜嫩。为了增加画 面的形式感, 采用对称式的构图, 签名亦采用中国传统 书画的签名方式, 采用中国传统记年的方式落款年月和 署名, 外加盖中国传统管刻的印章, 印章亦是笔者所刻 的管刻章。

\section{3. 《豚肉散记》水彩材料的选择与表现技法}

\section{1. 水彩工具材料的选择}

笔者手头的工具材料比较多样, 下面分别作详细的 介绍。画水彩的水彩颜料就有三种, 分别是国产合资的 温莎牌水彩颜料、日产的樱花牌水彩颜料以及法国进口 的温莎牌水彩颜料, 另外还有画国画的马利牌国画颜料 和画水粉的马利牌水粉颜料。看画面需要都是可以用的。 马利牌国画颜料也可以画水彩画, 但是等干了之后很难 清洗, 不好修改, 这是缺点也是优点。画水彩画肯定以 水彩颜料为主, 但是也不会排除其他颜料的运用, 以丰
富画面的表现力。

纸张的选择笔者主要用 300 克的进口阿诗纸和 300 克的国产宝虹牌水彩纸。因价格差异巨大, 而国产宝虹 纸又有不错的表现, 这里所用的都是国产 300 克宝虹水 彩纸, 这样也可以更好的体现出颜料的差异性, 比较颜 料之间的特点也更加的公平。

画笔的选择笔者之前都是选用扁平羊毫水彩笔, 因 其市面上难以买到合意的水彩扁平头水彩笔。所以笔者 改用中国传统羊毫毛笔作为画水彩的主要工具, 当然大 小可以多备几支，还有白描笔在勾线的时候也是不错的 选择。中国制的毛笔有非常丰富的表现力, 笔者用起来 得心应手, 另外再加扁平头水彩笔和羊毫刷子结合使用, 可以互补彼此的缺点。主体的刻画基本上都是用毛笔完 成的。羊毫刷子主要在平涂背景上用的较多。另外还值 得一提的是纸巾的运用, 因其吸水性强, 可以吸掉多余 的水分。也可以修形和提亮，在干的水彩画面涂上水， 然后用纸巾擦干, 可以得到比较亮的的形状, 在修形和 提亮上有不错的表现, 也算是一种不错的修饰工具。如 图 2

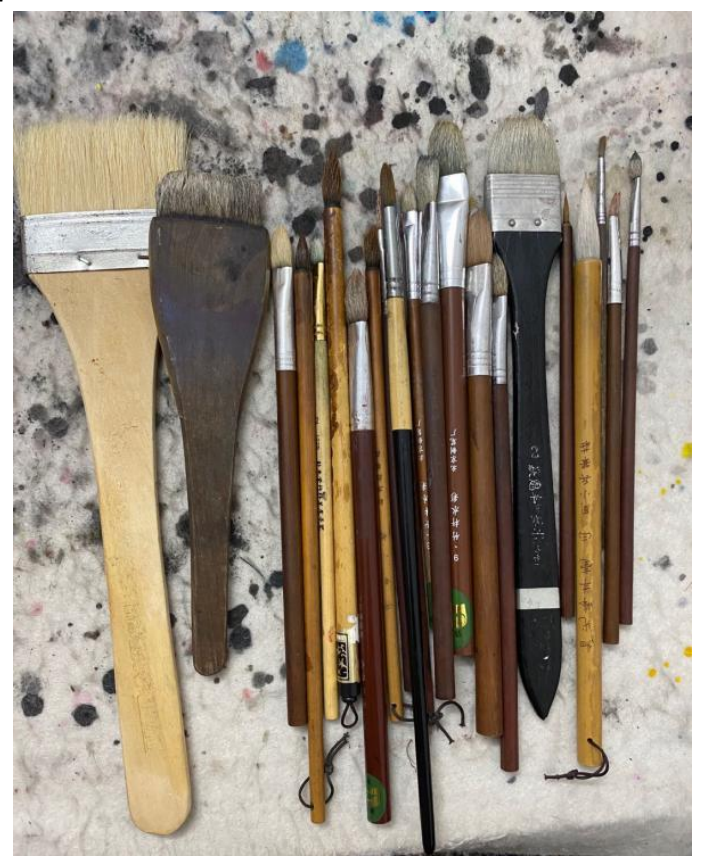

图 2 水彩画笔的选择

\section{2. 水彩画的步骤技法}

水彩画的表现技法丰富多样，各家各派各不相同， 在这里介绍笔者所采用的方法和最终画面效果展示 (如 图一), 画水彩画刚开始起稿时, 用的是 $2 \mathrm{~B}$ 铅笔起稿, 尽量少用橡皮檫, 以免损伤纸面。定稿之后就是上色, 一般开始采用湿画法, 先画猪肉的主体部分, 完成之后 再来平涂背景。为了有晕染开的效果, 一般在上色之前 会把纸打湿。

首先，将画纸放平在桌面上，用毛笔或刷子在局部 或整张铺上水, 也可以是颜色, 根据画面需要而定, 这 
一步需要注意的是要趁湿衔接, 因刚开始画的时候水份 多, 所以水份的控制尤为重要, 控制不好容易跑形, 或 者画面水份挥发过快不利于趁湿衔接, 衔接不上再画就 会显得生硬, 尤其是画比较大的画面, 这一切都要控制 好。笔者很喜欢笔触在画面自然晕染开的效果, 用了很 多的湿画法, 如猪肉红白相间的红色都是趁湿画上去的, 阴影也是趁着平涂背景时水份未干画上去的, 用这种方 法有意想不到的、有着很自然的晕染开来的效果, 很符 合阴影的感觉。

其次, 等画面完全干了之后再来塑造形体, 用笔要 轻松肯定, 同时也要注意颜色, 尽量一遍过, 少涂抹, 以免反复涂抹把底色带上来。主体猪肉部分所用颜料是 法国产进口温莎颜料, 色彩鲜艳, 反复涂抹也不容易脏。 也很符合猪肉那种鲜嫩的感觉, 有着不错的表现效果。 对于背景笔者想要一种平实的不抢眼的, 很平整的效果。 为此背景的颜料采用的是樱花牌水彩颜料, 樱花颜料颗 粒粗大, 塑造主体是难言满意, 但是拿来平涂背景时很 符合笔者的要求, 给人以很平实的感觉, 这种感觉正是 笔者所需要的。为此笔者还专门做过了研究实验, 以比 较这几种颜料所产生的差异。为了达到平实的效果, 采 用樱花颜料, 需要放平画板, 用大刷子平涂。纸张的背 面也可以喷些水, 可以减缓画面干燥的速度, 有助于画 面的衔接。

再次, 调整完成, 主要是在于一些细小的地方要调 整的, 如背景和主体之间的轮廓线往往会比较生硬, 需 要进一步调整, 让主体和背景更加自然协调。

最后, 落款签名就算完成了。至于落款笔者专门研 究了中国传统书法, 按照中国传统书法的落款方式进行 落款, 为作品增加亮点, 每个细节都精益求精。至于印 章, 笔者也为自己刻了几方管刻章, 但不是每张画都有 盖印。主要看创作时需要。

\section{3. 不同水彩颜料画背景的比较}

红花还需绿叶配, 初学者往往会更加注重主体的刻 画而忽略了背景的表现方式。背景的处理绝对不比主体 花的心思少, 笔者为了画出心仪的背景可花了不少心思。 一个好的背景应该是不突出抢眼, 而且能更加突出主体, 最后完成的画面效果给人感觉完整统一整体。为此必须 研究工具材料的表现技法，以便于画面有更好的表现力。 下面展示了五张不同牌子颜料背景的处理方式产生的 差异:

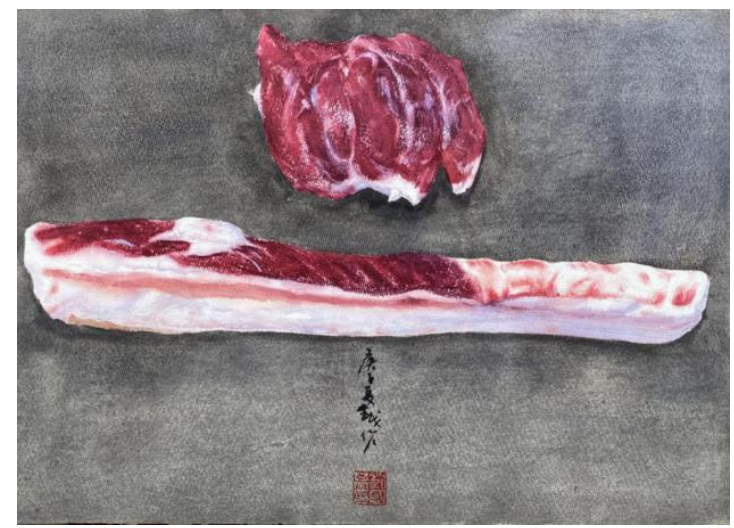

图 3 国产温莎的效果

图 3 这幅图背景部分采用了国产温莎的颜料, 从画 面效果可知这种颜料作为初学者可用, 但是对于专业画 家而言实难满意, 画面显得沉闷不通透。也很难平涂的 均匀平整。本来主体刻画还算满意, 背景破坏了画面的 整体感。

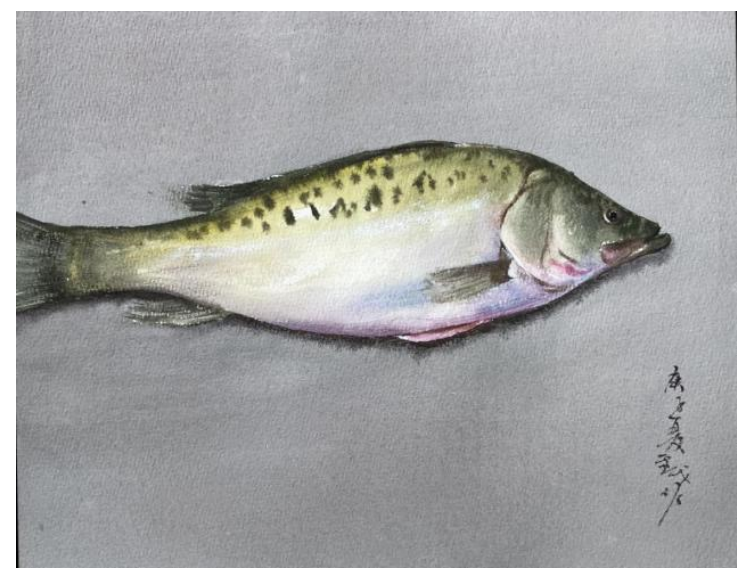

图 4 樱花的效果

图 4 这幅图用的是樱花水彩颜料平涂背景, 是笔者 想要的平实效果, 画面干净通透, 也显得更加整体, 《豚 肉散记》的背景全部采用樱花颜料平涂完成。但是樱花 颜料刻画主体则不是很令人满意。 


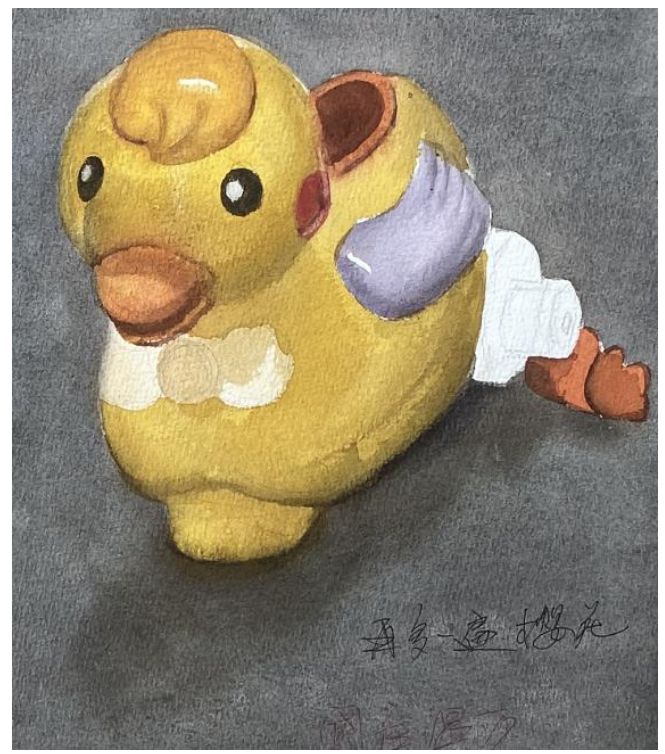

图 5 樱花加国产温莎的效果

图 5 这幅图的背景是用了国产温莎先画了一遍, 然 后再用樱花颜料叠第二遍。从图可知两种牌子的颜料混 在一起效果更是差，几乎结合了两者的缺点。

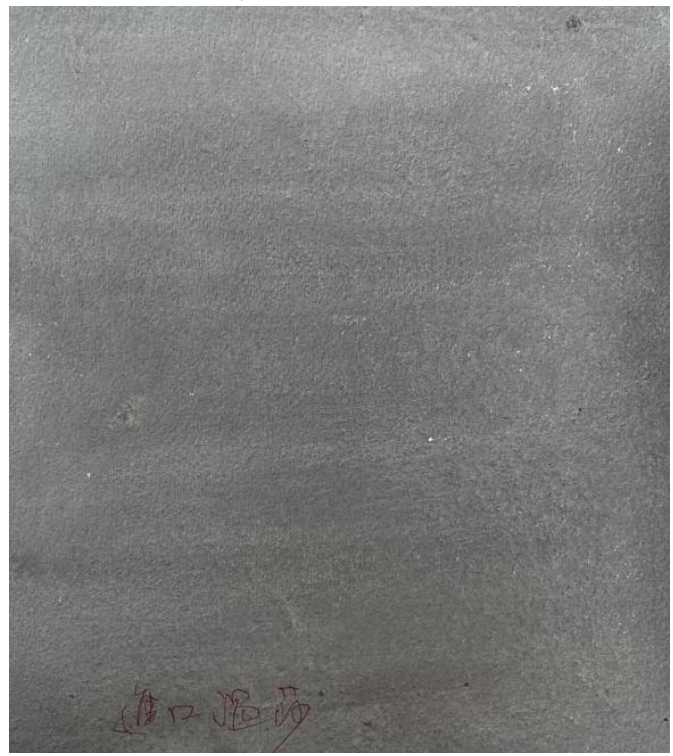

图 6 进口温莎的效果

图 6 这张用了法国进口温莎颜料, 从图可知, 法国 产的温莎颜料细淢, 效果干净通透, 刻画主体有着丰富 塑造与表现能力, 但是少了点浑厚效果, 并不是笔者想 要的平实感觉, 所以没有采用。

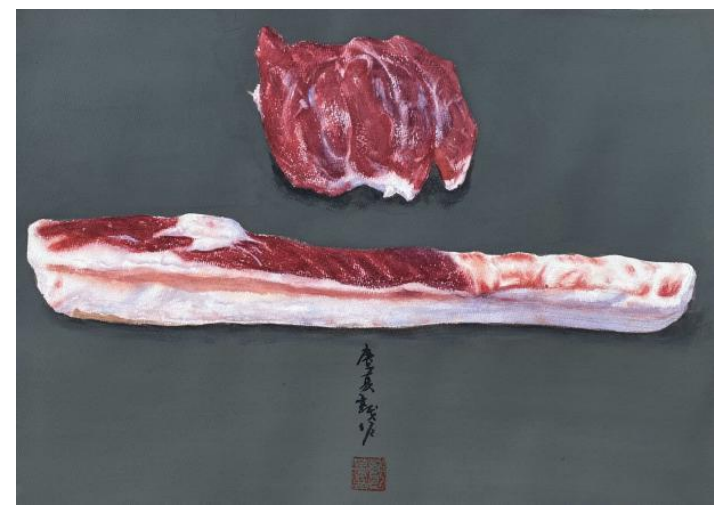

图 7 水粉颜料背景的效果

图 7 这张是在图三的基础上，因对图三的背景不满 意, 又重新用了马利牌水粉颜料平涂了背景, 因水粉颜 料覆盖能力强, 可以很好的把原先不满意的背景覆盖掉。 从图效果可知, 马利牌水粉颜料可以涂的比较平整, 缺 点是阴影的刻画会比较生硬，也会有种粉和不透气感， 但仍然比图三的效果要好，画面也更加干净整体。

另外还有大量的实验图片由于篇幅的限制不能一 一都在这里论述, 希望上述图片效果有抛砖引玉的作用。

\section{4. 结语}

所谓尺有所短寸有所长, 每种颜料都有各自的特性, 要充分发挥出它们的优点, 相对的就要避免它们的缺点, 才能表现出完美的画面。为此对所用的每种水彩颜料必 须熟悉, 必须通过反复练习才能掌握它们的特点并灵活 自如的运用。需要注意的是不要为了技法而技法, 只有 创作时所有的技法都恰如其分的运用到画面上并恰当 的表达出作者的思想情感与审美趣味，才是成功的技法。

\section{References}

[1] Ya,L. , (2004) Oil Painting, Watercolor by Schiller., Jilin Fine Arts Publishing House, Changchun

[2] Sargent, J. 2006, Sargent's Painting Collection, Tianjin Fine Arts Publishing House, Tianjin

[3] Wang Z.M., June 1985, Collection of Watercolor Paintings by Wang Zhaomin, Lingnan Fine Arts Publishing House, Guangzhou

[4] Long,H., (2011), The Noisy Structure: Longhu Works, Lingnan Fine Arts Publishing House, Guangzhou

[5] Baidu W., (2016), Pork.

https://wenku.baidu.com/view/ff4e53f1f71fb7360b4c2e3f5 727a5e9856a27dc.html,2016-11-28 